\title{
Research and Comparison of Solid Spallation Targets in Accelerator Driven System(ADS)
}

\author{
De-peng GUO \\ Nuclear and radiation safety center \\ Beijing, 100082, China \\ e-mail: guodepeng@chinansc.cn \\ www.chinansc.cn
}

Jing ZHAO*

Institute of Nuclear and New Energy Technology

Tsinghua University

Beijing, 100084, China

e-mail: zhao_jing@tsinghua.edu.cn

www.tsinghua.edu.cn

\begin{abstract}
The nuclear waste disposal problem is a difficulty for the world nuclear energy development. Accelerator driven sub-critical system (ADS) has been universally regarded as most efficiently in disposing the long-lived nuclear wastes. The spallation target is an important component in ADS as the coupling element of the accelerator system and the reactor system. Solid spallation target has several merits in ADS design and typical solid targets were compared in the paper.
\end{abstract} target

Keywords-Nuclear energy; Tansmutation; Ads; Spallation

\section{PREFACE}

\section{A. Nuclear Power Development in China}

Environmental pollution has become an important issue in the sustainable development of China's economy and society. Traditional coal-based thermal power generation has become the main source of air pollution, which seriously restricts the sustainable development of the national economy. The research and development of new energy and renewable energy becomes more and more important. Nuclear power, as a well-known clean energy source, has also become the focus of attention.

In 2005, China clearly put forward the strategic policy of actively developing nuclear power. According to the "Medium and Long Term Development Plan for Nuclear Power" promulgated by the State Council in 2007, the nuclear power installed capacity will reach $58 \mathrm{GW}$ by 2020 , and nuclear power under construction will be about $30 \mathrm{GW}$. The proportion of the total generating capacity will be increased to about 4\%. According to the analysis of the national medium and long-term energy development and prospects, the Chinese Academy of Engineering in the "2050 China's energy demand" advisory report pointed out that by 2050, China's nuclear power accounted for primary energy requirements increased to $12.5 \%$ (accounting for power installed capacity $20 \%$ ).

\author{
Fei XIE \\ Institute of Nuclear and New Energy Technology \\ Tsinghua University \\ Beijing, 100084, China
}

\section{B. Spent Fuel Disposal}

The rapid development of nuclear power will bring a key problem of spent fuel treatment. Spent fuels produced from a million kilo watt PWR nuclear power plants is approximate to $25 \mathrm{t}$ annually, including about 23.75 tons of recyclable $235 \mathrm{U}$ and $238 \mathrm{U}$, about 200 kilograms of plutonium, and about 1 ton of middle-lived and short-lived fission products(FPs), and also about 20 kilograms of minus actinides (Mas), and about 30 kilograms of long-lived fission product (LLFP).

With the installed capacity of China's pressurized water reactor nuclear power plant growth, the accumulation of nuclear waste will increase rapidly, if the nuclear power plant installed capacity reach of $8000 \sim 100$ million kilowatts in 2030, then accumulated fuel inventory will reach 2 million to 2.5 million tons, which contains Plutonium with the amount of 160 to 200 tons, MA of 16 to 20 tons, and LLFP of 24 to 30 tons.

In this case, the accelerator driven subcritical system (ADS) attracts the international attention as an effective means and advanced methods of disposing of spent fuels. ADS uses the international advanced separation and transmutation (Partition-Transmutation) strategy to deal with the nuclear waste. Through ADS the long-lived, high-radiation nuclides will be transmute into short-lived, low-level radioactive nuclides. After the transmutation treatment, the long-lived high-level radioactive waste can be reduced to the level of radioactivity of ordinary uranium ore in about 700 years. Compared with the closed-cycle model, the volume of nuclear waste which still need to be deeply buried in the ground after transmutation can be reduced more than five times. It can be found that the neutron transmutation method can not only reduce the amount and radioactivity of nuclear waste rapidly, but also improve the fuel efficiency. [1-6] 


\section{EFFECTIVE DISPOSAL OF NUCLEAR WASTE WITH ADS}

The accelerator-driven subcritical system (ADS) is composed of high-current proton accelerator, spallation target and sub-critical reactor. The proton beam generated by the accelerator bombarded the heavy metal sporadic target in the subcritical reactor. Neutron, for the sub-critical reactor to provide external neutrons to drive the fission reaction within the reactor. Principle of ADS is shown in Figure 1. Generally, the target is made of heavy metal in solid or in liquid state. The spallation neutron spectrum is harder and wider than the fast reactor (from thermal neutron to $\mathrm{GeV}$ ), allowing almost all of the threshold fission nuclides to be incinerated, and essentially all of the radioactive fission products can be transmuted.

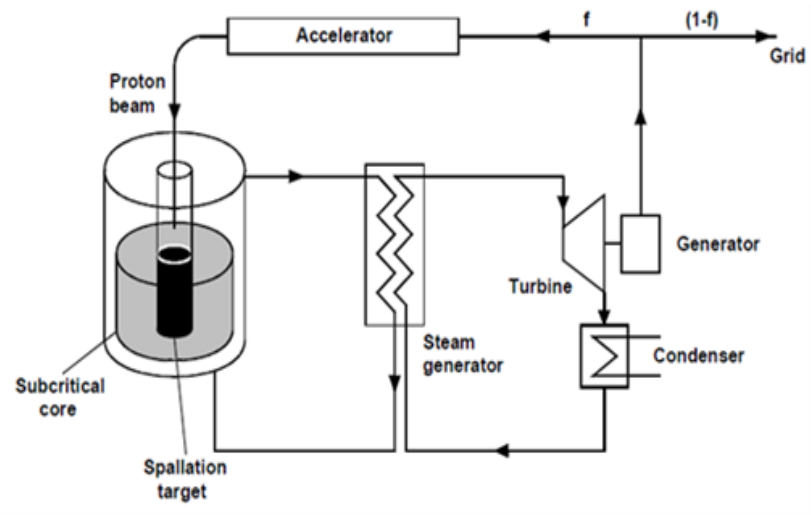

Figure 1. Schematic diagram of ADS

Compared with the critical reactor, ADS system has the following important features: Firstly, a powerful transmutation capability. The proton of energy $1 \mathrm{GeV}$ produces tens of neutrons plus tens of times magnification effect on the heavy metal target, so the ADS system has strong nuclear waste transmutation ability in principle. Secondly, high support ratio. Because the energy spectrum is harder and the more neutrons, an optimized ADS system can support a ratio of about 10 , while the fast reactor can only change the nuclear waste of about 2 to 5 PWRs due to the requirement of operational stability. The third, inherent security. Once the driving of the peripheral neutrons is cut off, the nuclear reactions in the subcritical system are stopped with inherent safety. Thus, in a variety of transmutation systems, ADS is a powerful nuclear waste transporters, and is a new technology approach for the sustainable development of nuclear fission energy.

\section{SPALLATION TARGETS IN ADS}

The spallation target is the very important component in the ADS system and a key component to distinguish the ADS from other reactor systems. It acts as the coupling of two systems, the accelerator system and the reactor system. Therefore, spallation target research is very important in ADS research. Generally, there are three main types of spallation targets which are often used in ADS systems.

First is the liquid target with windows. The material of the liquid target is usually heavy metals, such as $\mathrm{Hg}$, LBE, $\mathrm{Pb}$, etc. The design is relatively simple, but several kinds of heavy metal materials have their certain limitations, and the target window itself needs to consider its radiation and stress damage. Second is the liquid target without windows. The design is simple and does not need to take into account the target window performance, so the target system can withstand higher energy proton beam bombardment, which can increase the neutron yield. But its design and construction is of the higher difficulty, and the operation has instability. Third is the solid spallation target. Substituting liquid targets with solid metal targets can avoid the problem of high radioactivity and highly toxic decay product polonium 210, and strong corrosiveness and so on, and improves the practical operability of sub-critical stacks. And the solid target scheme has advantages in material compatibility and structural design.

\section{A. Maintaining the Integrity of the Specifications}

The template is used to format your paper and style the text. All margins, column widths, line spaces, and text fonts are prescribed; please do not alter them. You may note peculiarities. For example, the head margin in this template measures proportionately more than is customary. This measurement and others are deliberate, using specifications that anticipate your paper as one part of the entire proceedings, and not as an independent document. Please do not revise any of the current designations.

\section{RESEARCH OF SOLID SPALLATION TARGETS}

Research on solid spallation targets has been ongoing. The metal of Tungsten has the following excellent characteristics and is often used as a solid target material. [7]

- High n/p yield $(\sim 20)$,

- High atomic number (74),

- High density $\left(19.3 \mathrm{~g} \cdot \mathrm{cm}^{-3}\right)$,

- High melting point $\left(3400^{\circ} \mathrm{C}\right)$

- High thermal conductivity $\left(173 \mathrm{~W} \cdot \mathrm{m}^{-1} \cdot \mathrm{K}^{-1}\right)$

- Chemically inert, low corrosion

- Resistance to radiation damage( $(1$ year $)$

- Good availability and low price

Except the zirconium alloys are used as the material of the solid targets in the Swiss SING study, Tungsten are used as solid targets in other researches in several countries, such as in Germany, the United States, and the China Academy of Sciences. The current international designs of several major solid targets are compared in Table 1. [7-10]

TABLE I. SOLID TARGETS RESEARCH IN DIFFERENT COUNTRIES

\begin{tabular}{lllll}
\hline Schedule & 1 & 2 & 3 & 4 \\
\hline Country & Germany & Switzerland & USA & China \\
Project & AGATE & SING & & \\
Target Material & Tungsten & Zircaloy & Tungsten & Tungsten \\
Geometry & Segmented & Rods & Packed & Particle flow \\
& Plates & & Particles & \\
Beam Power(MW, & 1.2 & 11 & $\sim 10$ \\
\hline
\end{tabular}




\section{CONCLUSIONS}

Accelerator driven sub-critical system (ADS) has been universally regarded as most efficiently in disposing the long-lived nuclear wastes. The spallation target is an important component in ADS as the coupling element of the accelerator system and the reactor system. Solid spallation target deigns in the ADS system were summarized and compared in the paper. It can provide guidance for future research work on the ADS spallation target.

\section{ACKNOWLEDGMENT}

This work is supported by the National Natural Science Foundation of China (Grant No.11305092).

\section{REFERENCES}

[1] ZhaoZhixiang, Xia Haihong. Study on ADS and the sustainable development of nuclear energy [J]. China Nuclear Power, 2009, 2(3):202-211. (in Chinese)
[2] IAEA. Accelerator Driven Systems: Energy Generation and Transmutation of Nuclear Waste. Status Report. IAEA-TECDOC-985, November, 1997.

[3] OECD/NEA.Accelerator-driven Systems (ADS) and Fast Reactors $(F R)$ in Advanced Nuclear Fuel Cycles. Technical report (NEA-3109, 2002)

[4] Mansani L., et al.The European Lead-cooled EFIT Plant: An Industrial-scale Accelerator- Driven System for Minor Actinide Transmutation [J].Nuclear Technology, 2012, 180(2):241-263.

[5] Mansani L., et al.The European Lead-cooled EFIT Plant: An Industrial-scale Accelerator- Driven System for Minor Actinide Transmutation [J].Nuclear Technology, 2012, 180(2):241-263.

[6] Zhan Wenlong, XuHushan. Advanced Fission Energy Program - ADS Transmutation System [J]. Bulletin of Chinese Academy of Sciences, 2012, 27(3):375-381. (in Chinese)

[7] R.Nabbi, N.Shetty. Solid Tungsten Spallation Target for an Acceleratior-driven Transmutation System, Institute of Nuclear Fuel Cycle,RWTHArchen University.

[8] C. AMMREMAN, K. WOLOSHUN, et al. Conceptual Designs for a SpallationNeutron Target Constructed of a Helium-Cooled, Packed Bed of Tungsten Particles.Los Alamos National Laboratory.

[9] G.Heidenreich, Design of Solid Spallation Targets at PSI. (Paul ScherrerInstitut, 5232 Villigen PSI, Switzerland).

[10] Y.Xu, F.Kang, X.Sheng. Study on the Development of ADS and its Spallation Target ,Nuclear Science and Technology,2016,4(3),88-97. 